\title{
A Risk Assessment Method Based on OWGA Operator and Fuzzy DEMATEL Algorithm
}

\author{
Xiaohua Lin ${ }^{\mathrm{a}}$, Wenhua Jia ${ }^{\mathrm{b}}$ \\ aemail: zdlxh5001@163.com, bemail: wenhuajia@gmail.com, \\ School of Mechanical Engineering, Nanjing Institute of Technology, Nanjing, 211167,China
}

Keywords: failure mode and effect analysis (FMEA); risk assessment; triangular fuzzy number; ordered weighted geometric averaging operator; decision making trial and evaluation method (DEMATEL)

Abstract. For the deficiency of traditional failure mode and effects analysis ( FMEA) in solving risk ranking problem for related failure modes, this paper proposed a risk assessment method based on ordered weighted geometric averaging (OWGA) operator and fuzzy decision making trial and evaluation method (DEMATEL). Firstly, fuzzy evaluation of failure modes was carried out which is aggregated by OWGA operator, and the aggregated value was taken as the influence degree of failure cause effect on corresponding failure mode. Then, the initial direct influence matrix was constructed by DEMATEL method, normalize this matrix to compute the total influence matrix. According to the total influence matrix, the relation degree of each failure cause was calculated, based on which the failure risk assessment of the system can be carried out . The proposed method was applied in FMEA risk analysis of basic parts of metro car door system, the feasibility and effectiveness of the proposed method is verified by comparing with the results of the RPN method.

\section{Introduction}

Failure mode and effect analysis is a kind of bottom-up system security analysis method. FMEA analysis commonly used risk priority number (RPN) for quantitative analysis. First, we should determine the potential failure modes of the product or system, and then analyze the occurrence degree $(\mathrm{O})$, severity degree $(\mathrm{S})$ and detection degree of each failure mode. RPN number is the product of $\mathrm{O}, \mathrm{S}$ and $\mathrm{D}$, according to which we rank the failure mode and determine the priority of prevention and improvement measures.

However, the shortcomings of the RPN method are constantly emerging with the practical application, as follows: 1 ) The three risk factors $\mathrm{O}, \mathrm{S}, \mathrm{D}$ are regarded as equally important, ignoring the relative importance of $\mathrm{O}, \mathrm{S}$ and $\mathrm{D}$. In fact, different combination of $\mathrm{O}, \mathrm{S}$ and $\mathrm{D}$ could get the same RPN number. As the same RPN number means equivalent risk for corresponding failure modes, actually the potential risk may differ. 2) Evaluation of the three risk factors $\mathrm{O}, \mathrm{S}, \mathrm{D}$ is usually qualitative, fuzzy and uncertain, as the traditional method has limitations in dealing with fuzzy information, which lead to the restricted application of FMEA. 3) In many cases, there are many related failure causes for the failure modes of product or system, this correlation is not considered in traditional RPN method, which leads to the deviation of analysis results.

Due to the shortcomings of the traditional RPN methods, experts and scholars have made some improvements. Zhou [1] applied fuzzy set theory to calculate the fuzzy RPN values of failure modes, grey theory to calculate the grey correlation coefficient of failure modes, the ranking of failure modes is determined by the fuzzy RPN and the grey correlation coefficients. Lolli [2]introduces a multi-criteria decision-making method to solve the related FMEA problem. But, it is very difficult to obtain the accurate information when used to complex products or systems. Emovon [3] proposed a ranking method combining VIKOR algorithm and the compromise-based mean algorithm, which can not suitably represent uncertain information. Rafie [4] proposed a fuzzy FMEA method, in which the severity degree and detection degree are obtained by fuzzy rules, as the occurrence degree is trained by artificial neural network, so this method needs a large number of samples.

Although these methods can partly overcome the defects of traditional FMEA, but its application 
condition is relatively harsh, the computation is complicated. In particular, there is not a simple and efficient method for ranking problem of related failure modes in fuzzy environment.

Therefore, this paper will propose a hybrid method based on ordered weighted geometric averaging operator (OWGA) and fuzzy DEMATEL algorithm, which can not only break through the limitations of the traditional RPN method, but also can deal with the related issues between failure modes in fuzzy environment. The aggregated values are taken as the direct influence degree between failure modes, then the relation degree of each failure cause is calculated for ranking. Finally, the feasibility and effectiveness of the proposed method is verified by the application of the FMEA analysis of base parts of metro car door system.

\section{OWGA operator}

Ordered weighted geometric averaging operator (OWGA) is an effective method to aggregate data information. OWGA operator can get the optimal weight vector which is based on the ranking of vector [5].

Definition 1: Given $R^{+}$as the positive real number set, $G_{W}: R^{+n} \rightarrow R^{+}$, there is $G_{W}\left(a_{1}, a_{2}, \cdots a_{n}\right)=\prod_{j=1}^{n} b_{j}^{w_{j}}$. Where, $W=\left(w_{1}, w_{2}, \cdots w_{n}\right)^{T}$ is the exponentially weighted vectors related with $G$, which satisfy $\sum_{j=1}^{n} w_{j}=1$ and $w_{j} \in[0,1] . b_{j}$ is the jth bigger number in data set $\left(a_{1}, a_{2}, \cdots a_{n}\right)$, the function $G$ is called ordered weighted geometric averaging operator, abbreviated as OWGA. For fuzzy number to be processed, OWGA operator will be as follows:

$$
\tilde{G}_{W}\left(\tilde{a}_{1}, \tilde{a}_{2}, \cdots \tilde{a}_{n}\right)=\sum_{j=1}^{n} \tilde{b}_{j}^{w_{j}}
$$

Definition 2: Set $W=\left(w_{1}, w_{2}, \cdots w_{n}\right)^{T}$ as the weight vector of OWGA operator, and define $O(W)=\frac{1}{n-1} \sum_{j=1}^{n}(n-j) w_{j}$ as the orness measure for the operator. OWGA weight vector can be determined by a given subjective decision coefficient and the maximum entropy, refer to literature [6].

\section{Fuzzy DEMATEL}

In this paper, DEMATEL and the fuzzy theory is combined to analyze the relationships between elements in FMEA system. The steps of the fuzzy DEMATEL method are as follows:

Step 1: Construct the initial direct influence fuzzy matrix $\widetilde{Z}=\left[\tilde{Z}_{i j}\right]_{n \times n}$. Where, $\tilde{z}_{i j}=\left(z_{i j 1}, z_{i j 2}, z_{i j 3}\right)$ is the influence strength for element $\mathrm{i}$ effect on element $\mathrm{j}$ in the form of triangular fuzzy number.

Step 2: Construct the normalized direct influence fuzzy matrix.

$$
\tilde{X}=\tilde{Z} / r, \quad r=\max _{1 \leq i \leq n}\left(\sum_{j=1}^{n} z_{i j 3}\right)
$$

Step 3: Compute the total influence fuzzy matrix matrix $\widetilde{\boldsymbol{T}}=\left(\tilde{t}_{i j}\right)_{n \times n}$ as follows:

$$
\widetilde{T}=\lim _{k \rightarrow \infty}\left(\widetilde{X}^{1} \oplus \widetilde{X}^{2} \oplus \ldots \oplus \widetilde{X}^{k}\right)=\widetilde{X}(\tilde{I}-\widetilde{X})^{-1}
$$

Where, divide the matrix $\widetilde{\boldsymbol{T}}$ into three matrix, namely, $T_{1}=\left[t_{i j 1}\right]_{n \times n}=X_{1}\left(I-X_{1}\right)^{-1}$, $T_{2}=\left[t_{i j 2}\right]_{n \times n}=X_{2}\left(I-X_{2}\right)^{-1}, \quad T_{3}=\left[t_{i j 3}\right]_{n \times n}=X_{3}\left(I-X_{3}\right)^{-1}, \quad$ and $\quad \boldsymbol{I}$ is a unit matrix.

Step 4: Determine the causal relationship between elements. $\tilde{D}_{i}$ and $\tilde{R}_{i}$ are the sum of row and column elements, respectively. $\quad \tilde{P}_{i}=\tilde{D}_{i}+\tilde{R}_{i}$ and $\tilde{C}_{i}=\tilde{D}_{i}-\tilde{R}_{i}$ represent the predominance degree and relation degree of corresponding element, respectively. Predominance degree shows the importance of element in the whole system, and relation degree shows the role of element in the system. If $C_{i}>0$, the element $\mathrm{i}$ is classified as reason element. Contrarily, the element $\mathrm{i}$ is classified 
as result element. The bigger the relation degree of failure cause, the higher the risk.

\section{Hybrid FMEA Model}

This paper apply the OWGA aggregated values as the input data of the fuzzy DEMATE algorithm, to construct the initial direct influence matrix for FMEA system. Fuzzy DEMATEL method is performed to get the relation degree of the FMEA element, according to which we rank the failure causes. The specific steps are as follows:

Step 1: List all the potential failure modes. According to historical data and their experience, FMEA experts list the potential failure modes for the product or system.

Step 2: List all the reasons caused the failure modes. FMEA experts will judge how the system failure occurs, and what type of the failure modes are, these analysis results will be listed in the FMEA table.

Step 3: Evaluate the risk of failure modes. Define the fuzzy evaluation terms for evaluation factors of failure mode (including $\mathrm{O}, \mathrm{S}$ and $\mathrm{D}$ ), as shown in table 1. Experts evaluate occurrence degree, severity degree and detection degree of each failure mode in fuzzy language, the evaluation information will be translated into triangular fuzzy number according to table 1 and then averaged.

Step 4: Calculate the OWGA weights. Select $\alpha$ as the subjective decision coefficient, $\alpha \in[0,1]$. Calculate OWGA weights referring to literature [6].

Step 5: Calculate the OWGA values. According to the information obtained in steps 3 and 4, we compute the OWGA value for each failure cause on its corresponding failure mode per formula (1).

Step 6: Implement fuzzy DEMATEL method to take FMEA analysis.

1) construct the initial fuzzy influence matrix of FMEA system, the elements of the matrix are the OWGA aggregated values obtained in step 5.

2) compute the normalized direct influence fuzzy matrix per formula (2).

3) compute the total influence matrix per formula (3).

4) calculate predominance degree and relation degree for failure modes and failure causes.

Step 7: assess the failure risk for FMEA system according to the relation degree of failure causes, and rank the failure causes.

Step 8: if the risk is greater than the threshold, the corresponding failure mode must execute improvement measures, and return to step 3 to reevaluate.

Step 9: if all the risk are less than the threshold, finish the FMEA analysis, and output the results.

\section{Case Study}

The proposed method is applied to FMEA analysis of the basic parts of metro car door system. The traditional FMEA analysis is shown in table 2. The evaluation information of failure modes is shown in table 3, which is determined by FMEA team composed of 11 experts. The subjective decision coefficient is taken as $\alpha=0.7$ for this application, then we computed the OWGA weights, namely $\omega_{1}=0.554 \omega_{2}=0.292 \omega_{3}=0.154$. The evaluation information is aggregated by OWGA operator, and the results are shown in column 7 of table 3. The initial influence matrix of FMEA system can be established by the OWGA values, then we applied fuzzy DEMATEL algorithm to obtain relation degree of each failure cause (results are shown in table 3 ).

The descending orders of failure causes by traditional RPN method and by fuzzy OWGA-DEMATEL method are shown in table 2 and table 3, respectively. We can get following conclusion by comparing the two descending orders:

1) CF 10 is with maximum risk by RPN method, and CF 2 is by the proposed method. The reason is that CF2 will lead to failure modes FM1, FM3 and FM4, simultaneously, although CF10 has higher RPN value, but in fact the risk of CF2 is higher.

2) the RPN value of CF1 $(3 \times 8 \times 3)$ and CF6 $(2 \times 6 \times 6)$ is the same 72 , but the fuzzy OWGA-DEMATEL method can distinguish these two, and the results show that the risk of CF1 is 
Tab.1 Triangular fuzzy values corresponding to the traditional evaluation terms

\begin{tabular}{lllllllll}
\hline \multicolumn{2}{c}{ Evaluation terms } & \multicolumn{2}{c}{ Occurrence } & \multicolumn{2}{c}{ Severity } & \multicolumn{2}{c}{ Detection } \\
\hline $\begin{array}{l}\text { fuzzy } \\
\text { semantcs }\end{array}$ & $\begin{array}{l}\text { Crisp } \\
\text { values }\end{array}$ & $\begin{array}{l}\text { Fuzzy } \\
\text { evaluation }\end{array}$ & $\begin{array}{l}\text { Occurrence } \\
\text { probability }\end{array}$ & Fuzzy evaluation & $\begin{array}{l}\text { Traditional } \\
\text { evaluation }\end{array}$ & $\begin{array}{l}\text { Fuzzy } \\
\text { evaluation }\end{array}$ & $\begin{array}{l}\text { Fuzzy } \\
\text { Detection } \\
\text { probability } \\
(\%)\end{array}$ \\
\hline Very low & 1 & Rarely & $<1: 20000$ & No influence & slight & very easily & $86-100$ & $(0,0,1)$ \\
low & 2,3 & occasionally & $1: 20000 ~ 1: 10000$ & Affect & mild & easily & $66-85$ & $(0,1,3)$ \\
Mediate low & 4 & sometimes & $1: 10000 ! 1: 2000$ & Minor defect & light & Medium easy & $56-65$ & $(1,3,5)$ \\
mediate & 5,6 & Often & $1: 2000 ~ 1: 200$ & A little big & mediate & possibly & $46-55$ & $(3,5,7)$ \\
Mediate high & 7 & More often & $1: 200 ~ 1: 20$ & Great defect & heavy & Medium hard & $36-45$ & $(5,7,9)$ \\
High & 8,9 & frequently & $1: 20 ~ 1: 10$ & Function scrap & severe & hardly & $16-35$ & $(7,9,10)$ \\
Very high & 10 & inevitably & $>1: 10$ & Threat to & deadly & unlikely & $0-15$ & $(9,10,10)$ \\
\hline
\end{tabular}

Tab.2 Potential failure modes and failure causes of basic components of metro car door

\begin{tabular}{clllllll||llllllll}
\hline No. & $\begin{array}{l}\text { Failure } \\
\text { modes }\end{array}$ & $\begin{array}{l}\text { Failure } \\
\text { causes }\end{array}$ & O & S & D & RPN & $\begin{array}{c}\text { Risk } \\
\text { rank }\end{array}$ & No. & $\begin{array}{l}\text { Failure } \\
\text { modes }\end{array}$ & $\begin{array}{l}\text { Failure } \\
\text { causes }\end{array}$ & O & S & D & RPN & $\begin{array}{c}\text { Risk } \\
\text { rank }\end{array}$ \\
\hline 1 & FM1 & CF1 & 3 & 8 & 3 & 72 & 4 & 8 & FM6 & CF6 & 2 & 6 & 6 & 72 & 4 \\
2 & FM1 & CF2 & 4 & 9 & 5 & 180 & 2 & 9 & FM6 & CF7 & 2 & 6 & 3 & 36 & 7 \\
3 & FM2 & CF3 & 2 & 5 & 3 & 30 & 10 & 10 & FM7 & CF8 & 3 & 4 & 2 & 24 & 11 \\
4 & FM3 & CF2 & 4 & 5 & 5 & 100 & 2 & 11 & FM8 & CF9 & 3 & 6 & 2 & 36 & 7 \\
5 & FM3 & CF4 & 2 & 5 & 5 & 50 & 6 & 12 & FM9 & CF10 & 5 & 9 & 5 & 225 & 1 \\
6 & FM4 & CF2 & 4 & 7 & 5 & 140 & 2 & 13 & FM10 & CF11 & 2 & 2 & 4 & 16 & 12 \\
7 & FM5 & CF5 & 4 & 9 & 4 & 144 & 3 & 14 & FM11 & CF12 & 3 & 4 & 3 & 36 & 7 \\
\hline
\end{tabular}

Tab.3 Fuzzy evaluation data for safety analysis of basic components of metro car door

\begin{tabular}{lllcccccc}
\hline No. & $\begin{array}{l}\text { Failure } \\
\text { modes }\end{array}$ & $\begin{array}{l}\text { Failur } \\
\text { e }\end{array}$ & O & S & D & OWGA value & Relation degree & rank \\
\hline 1 & FM1 & CF1 & $(0.8,2.7,4.7)$ & $(6.3,8.3,9.7)$ & $(0.7,2.3,4.3)$ & $(4.656,5.703,6.344)$ & $(0.242,0.296,0.330)$ & 4 \\
2 & FM1 & CF2 & $(1.6,3.6,5.6)$ & $(6.7,8.7,9.8)$ & $(3.5,5.5,7.5)$ & $(5.385,6.178,6.646)$ & $(0.769,0.902,1.000)$ & 1 \\
3 & FM2 & CF3 & $(0.4,1.6,3.6)$ & $(3.5,5.5,7.5)$ & $(0.8,2.5,4.5)$ & $(3.807,4.953,5.823)$ & $(0.188,0.247,0.292)$ & 10 \\
4 & FM3 & CF2 & $(1.6,3.6,5.6)$ & $(3.0,5.0,7.0)$ & $(3.5,5.5,7.5)$ & $(4.455,5.389,6.122)$ & $(0.769,0.902,1.000)$ & 1 \\
5 & FM3 & CF4 & $(0.4,1.6,3.6)$ & $(3.0,5.0,7.0)$ & $(3.5,5.5,7.5)$ & $(4.248,5.246,6.036)$ & $(0.225,0.277,0.318)$ & 6 \\
6 & FM4 & CF2 & $(1.6,3.6,5.6)$ & $(5.0,7.0,9.0)$ & $(3.5,5.5,7.5)$ & $(4.956,5.802,6.483)$ & $(0.769,0.902,1.000)$ & 1 \\
7 & FM5 & CF5 & $(1.9,3.9,5.9)$ & $(6.7,8.7,9.8)$ & $(1.7,3.7,5.7)$ & $(5.160,6.026,6.528)$ & $(0.268,0.313,0.339)$ & 3 \\
8 & FM6 & CF6 & $(0.7,2.3,4.3)$ & $(4.0,6.0,8.0)$ & $(4.0,6.0,8.0)$ & $(4.295,5.291,6.073)$ & $(0.233,0.285,0.325)$ & 5 \\
9 & FM6 & CF7 & $(0.8,2.4,4.4)$ & $(4.0,6.0,8.0)$ & $(1.0,3.0,5.0)$ & $(4.122,5.221,6.021)$ & $(0.217,0.275,0.316)$ & 7 \\
10 & FM7 & CF8 & $(1.0,3.0,5.0)$ & $(1.7,3.7,5.7)$ & $(0.4,1.7,3.7)$ & $(3.210,4.528,5.446)$ & $(0.167,0.235,0.283)$ & 11 \\
11 & FM8 & CF9 & $(1.0,3.0,5.0)$ & $(3.6,5.6,7.6)$ & $(1.0,3.0,5.0)$ & $(4.033,5.160,5.957)$ & $(0.210,0.268,0.309)$ & 8 \\
12 & FM9 & CF10 & $(1.5,3.5,5.5)$ & $(6.5,8.5,9.5)$ & $(3.5,5.5,7.5)$ & $(5.327,6.131,6.582)$ & $(0.277,0.318,0.342)$ & 2 \\
13 & FM10 & CF11 & $(0.9,2.7,4.7)$ & $(0.4,1.7,3.7)$ & $(1.6,3.6,5.6)$ & $(3.136,4.455,5.392)$ & $(0.153,0.221,0.270)$ & 12 \\
14 & FM11 & CF12 & $(0.9,2.7,4.7)$ & $(2.8,4.8,6.8)$ & $(1.3,3.3,5.3)$ & $(3.833,4.967,5.789)$ & $(0.195,0.258,0.301)$ & 9 \\
\hline
\end{tabular}

higher. This is due to the ordered weights for the three risk factors when the latter is used to aggregate the evaluation information, while the former does not take into account the relative importance of the risk factors.

3) CF7 $(2 \times 6 \times 3)$, CF9 $(3 \times 6 \times 2)$ and CF12 $(3 \times 4 \times 3)$ are deemed the same risk as they have the same RPN value, but they can be distinguished by fuzzy OWGA-DEMATEL method. There are two reasons for the different results: a) the ordered weighted aggregation of the information; b) fuzzy representation of evaluation information, fuzzy qualitative semantics can fully convey the uncertainty of the information, thus failure modes with same crisp RPN value can be distinguished.

4) In traditional method, the repetition rate of RPN values is a bit high, which lead to the same risk ranking order for a number of failure causes. However, there is no repetition of the ranking values (namely, relation degree ) by the proposed method, which provides decision makers a more explicit reference when they develop failure prevention measures. 


\section{Conclusion}

We can obtain the risk ranking of relative failure modes in fuzzy environment by the proposed method, and its advantages are the following: 1 ) reduce the repetition rate of risk evaluation values; 2) dynamically allocate the weights of the three factors (O, S and $\mathrm{D})$; 3) this method is very effective for analyzing internal-related system, where the influence degree between internal elements of the system are determined; 4) the analysis result is more close to the reality since the fuzzy theory is used to represent the failure information in FMEA.

\section{Acknowledgement}

The authors would like to thank the National Natural Science Foundation of China (No.51405222) and Imported Talent Scientific Research Foundation of Nanjing Institute of Technology (No.YKJ201301)

\section{References}

[1]Zhou Q, Thai V V. Fuzzy and grey theories in failure mode and effect analysis for tanker equipment failure prediction[J]. Safety Science, 2016,( 83):74-79.

[2]Lolli F, Ishizaka A, Gamberini R, et al. FlowSort-GDSS - A novel group multi-criteria decision support system for sorting problems with application to FMEA[J]. Expert Systems with Applications, 2015, 42(17):6342-6349.

[3]Emovon I, Norman R A, Alan J M, et al. An integrated multicriteria decision making methodology using compromise solution methods for prioritising risk of marine machinery systems[J]. Ocean Engineering, 2015, 105(10):92-103.

[4]Rafie M, Namin F S. Prediction of subsidence risk by FMEA using artificial neural network and fuzzy inference system[J]. International Journal of Mining Science \& Technology, 2015, 25(4):655-663.

[5] Chang, K.H., Chang, Y.C., Wen, T.C., Cheng, C.H., 2012. An innovative approach integrating 2-tuple and LOWGA operators in process failure mode and effects analysis. Int. J. Innov. Comput. Inf. Control 8 (1), 747-761.

[6] Fuller, R., Majlender, P., 2001. An analytic approach for obtaining maximal entropy OWA operator weights. Fuzzy Sets Syst. 124 (1), 53-57. 